Gewebeschichten, z. B. Fissidens decipiens, Polytrichum commune etc., aber bei der Mehrzahl erhält man die Cellulosereaction erst nach Vorbehandlung der Schnitte mit Chromsäure oder Schulze'schel' Mischung.

3. Pectinstoffe sind stets in der Zellhant der Moose vorhanden.

Arbeiten des botanischen Institutes der k. k. deutschen Universität Prag. XVII.

\title{
Drei Carpelle bei einer Umbellifere (Cryptotaenia canadensis).
}

Von Dr. Jos. Rompel S. J. (Prag).

(Mit 3 Figuren.)

Im Vergleiche zu den schwankenderen Verhältnissen bei anderen Pflanzenfamilien ist es auffallend, dass bei der Familie der Umbelliferen sich die Zweizahl der Fruchtblätter als recht constante Erscheinung erweist. Man sollte in Anbetracht der Fünfzahl der übrigen Blütenquirle und in Anbetracht des ausserordentlichen Artenreichthums der Familie ein häufigeres Auftreten von mehr als 2 Fruchtblättern erwarten, als es thatsächlich der Fall zu sein scheint oder wenigstens beobachtet worden ist. Die bisher namhaft gemachten Fälle von mehr als 2 Carpellen verschwinden so sehr gegenüber der grossen Artenzahl der Familie, dass man sie mit Eichler (Blütendiagramme, II. S. 407) nur als "zufällige Ausnahme" bezeichnen muss.

Wohl die vollständigste Zusammenstellung der beobachteten Fälle findet sich in Penzig's Teratologie. Hier wird von 18 Arten angegeben, dass gelegentlich mehr als 2 Carpelle beobachtet wurden. Dabei ist aber zu bemerken, dass für eine ganze Reihe dieser Arten diese Beobachtung nur an der centralen Blüte eines Döldchens gemacht wurde, nicht auch an seitlichen Blüten, dass ferner bei anderen Arten eine solche Vermehrung nur statthatte bei gleichzeitiger Vergrünung der Blüte.

Es dürfte zunächst nicht unnütz sein, diese 18 Arten durch einige andere zu vermehren, an welchen schon früher Polyphyllie des Gynöceums beobachtet wurde, die aber Penzig entgangen sind.

In dem Umbelliferenband von Reichenbach's Icones Florae Germanicae (Vol. XXI) zeigt:

Tafel 13, Fig. 16 Apium graveolens mit 3 Fruchtblättern, deren allerdings in der Erklärung nicht Erwähnung geschieht;

Tafel 17, Fig. 18 Petroselinum Thorei mit der Erklärung: "Flos valde monstrosus quasi calice duplici ac stylis quaternis";

Tafel 20, Fig. 11 Aegopodium Podagraria mit der Bemerkung: "Fructus tricoccus monstrosus". 
In Hoffmann's Genera Umbelliforarum findet sich bei Scandix (Wylia) australis die Bemerkung: „Styli, sed rarius, etiam tres occurrunt." zuzählen.

Diese 4 Arten sind also den von Penzig angeführten zu-

In mehrfacher Hinsicht ist die von mir beobachtete Polyphyllie des Gynöceums der nordamerikanischen Umbellifere Cryptotaenia canadensis DC. von Interesse. Die Planze, von der ich sowohl im Sommer des vorigen als auch im Sommer dieses Jahres junge Fruchtstadien beobachten konnte, befindet sich im botanischen Garten zu Prag. Dem Habitus nach lässt sie sich unter den einheimischen Umbelliferen wohl am besten mit Aegopodium Podagraria vergleichen, welcher Pflanze sie gewiss auch im Systeme nicht ferne steht.

Bevor auf die Carpellzahl eingegangen wird, ist eine anatomische Eigenthümlichkeit zu erwähnen, welche für diese Species ganz constant allen Früchten zukommt, sonst aber unter den Umbelliferen meines Wissens nirgends beobachtet ist. Wie bekannt sind für das Pericarp der Umbelliferen fünf Gefässbündelstränge charakteristisch. Betrachtet man nun einen Quersehnitt der CryptataeniaFrucht, so findet man sieben Gefässbündel im Pericarp jeder Theilfrucht, dazu noch den Carpophor an der Commissur. Fig. 1 stellt einen solchen Querschnitt. dar. Die sieben Gefässbündel in jeder Theilfruchtwand, der Carpophor und das Gefässbündel der Rhaphe sind durch Punktirung kenntlich. Die 1-4 kleinen Kreise ansserhalb jedes Gefässbündels, sowie die tangential gestreckten, kranzförmig dem Endocarp eingelagerten Gebilde sind Querschnitte von Secretgängen, mit denen

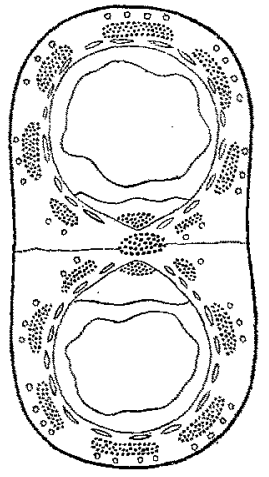

Fig. 1. - Vergr. $25 / 1$. wir uns hier nicht weiter befassen. So ungefähr ist das Bild beschaffen, das der Querschnitt einer normalen, halbreifen Frucht bei schwacher Vergrösserung dem Beschaner darbietet; anch die Früchte von Pfanzen, welche nicht cultivirt, sondern aus der Heimat dieser Art gesammelt worden waren, verhielten sich genau ebenso.

Das Exemplar der Pflanze im botanischen Garten zu Prag zeigt weiterhin eine gewisse Hinneigung zur Ausbildung von d rei Carpellen. Im Herbst 1894 konnte ich ungefähr 100 junge bis halbreife Früchto daraufhin untersuchen. Dieselben waren im Spätsommer ohne Auswahl zum Zwecke einer anderen Untersuchung in Alkohol gegeben worden. Bs fanden sich nun 8 Früchte, welche ein 3. Fruchtblatt in mehr oder weniger deutlicher Ausbildung besassen. Bei diesen waren die 3 Griffel überall dentlich vorhanden, doch der eine zuweilen etwas schmächtiger ausgebildet. Der Fruchtknoten aber zeigte das 3. Carpell in der verschiedensten Weise ausgebildet. Es fanden sich alle Uebergänge von einer zwischen die beiden normal vorhandenen 
Fruchtblätter eingeschalteten dünnen Lamelle bis zur völlig gleichen Ausbildung der 3 Carpelle. Fig. 2 zeigt das 3. Fruchtblatt nur als schwache Anlage mit 3 Gefässbünden, während die zwei anderen Pericarpe die Normalzahl der Gefässbündel besitzen (Secretgänge nicht eingezeichnet). Fig. 3 lässt kaum einen Unterschied in den drei Fruchtblättern erkennen, nur sind sie in der Entwicklung der Fruchthöhle nicht gleich weit vorgeschritten (mit 1,2,3 bezeichnet). Es ist aber bemerkenswerth, dass nicht in jeder Theilfrucht sieben, sondern in 2 Theilfrüchten fünf und in der dritten 6 Gefässbündel im Pericarp ausgebildet sind. Ob wohl die Siebenzahl der Gefässbündel einer typischen Theil-

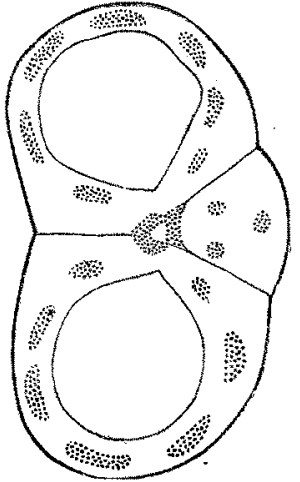

Fig. 2. - Vergr. 25/1.

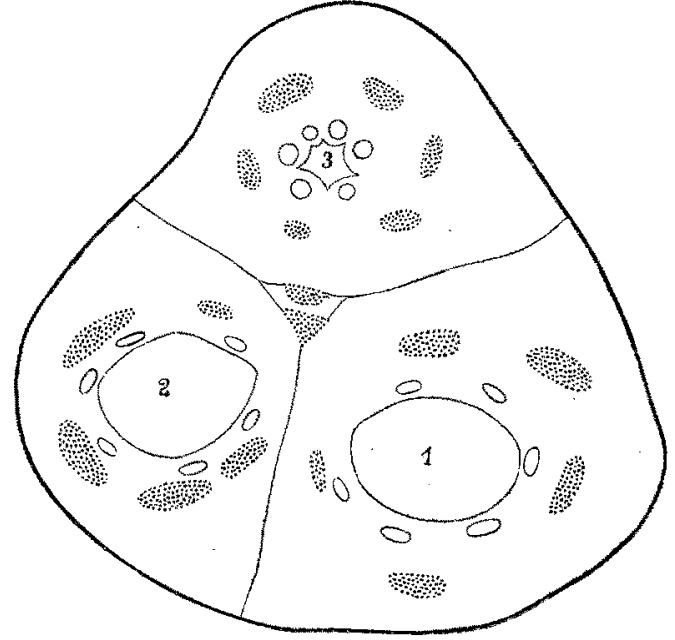

Fig. 3. - Vergr. 50/1.

frucht mit der Neigung zur Ausbildung von 3 Carpellen in Verbindung steht? Diese Frage muss unentschieden bleiben, um so mehr, da eine dreigriffelige Frucht ganz oben im Fruchtknoten keine Spur eines dritten Carpells mehr erkennen liess, aber in der einen Hälfte jetzt sogar 8 Gefässbündel aufwies. Ferner ist noch die Thatsache zu beachten, dass ich an 5 Exemplaren der Pflanze, welche sich reichlich mit Früchten versehen im Herbar des botanischen Institutes zu Prag vorfinden, keine einzige Frucht mit 3 Carpellen entdeckte. Demnach ist die Hinneigung zu 3 Carpellen nicht eine Eigenthümlichkeit der Pflanze im allgemeinen, sondern eben nur des Individuums im botanischen Garten zu Prag.

Im Sommer laufenden Jahres konnte ich mich nun überzengen, dass die Pflanze - dasselbe Individuum - sich in der Ausbildung dreier Carpelle treu bleibt. Von ungefähr 400 Früchten, welche jch ohne Auswahl hernahm und durchmusterte, zeigten sich widerum häufig genng solche mit 3 Frucbtblättern, ungefähr $3 \%$ der nnter- 
suchten Früchte. Ich muss hinzufügen, dass ich diesmal auch eine Frucht mit vier und eine andere mit fün $\mathrm{f}$ Carpellen fand. Die letztere zeigte alle Griffel gleichmässig ausgebildet, gehörte einer seitlichen und nicht einer centralen Bläte an, und besass, wie zu erwarten. war, eine derartige Anordnung des Gynöceums und Andröceums, dass die 5 Fruchtblätter mit den 5 Staubblättern -alternirten. Bei den Früchten mit 3 Carpellen war die Stellung des unpaaren Fruchtblattes nicht bestimmt, sondern in mehrfacher Weise wechselnd. Auch diese Früchte gehörten meistens seitlichen, ganz selten centralen Blüten an. - Zu bemerken ist noch, dass auch bei normal zweifächerigen Fruchtknoten das eine Fach ziemlich hänfig abortirt. Dadurch kommt eine eigenthümliche, oft sehr starke Krümmung der ganzen Frucht zustande, wobei das abortirende, nicht mehr in die Länge wachsende Fruchtblatt natürlich die Concarseite einnimmt. Das Gattungssynonym Cyrtospermum (xverós gekrümmt) wurde durch diese Krümmung veranlasst. ')

Prag, den 10. Juli 1895.

\section{Beitrag zur Flora von Griechenland. \\ Von Dr. E. von Halácsy (Wien).}

(Fortsetzung. ${ }^{2}$ )

81. Bupleurum trichopodum Bois. et Sprun. in Ann. Sc. nat. 1844 , p. 145 .

Insel Salamis (Reiser).

82. Scaligeria cretica Urv. Enum. p. 31 (1822) sub Bunio; Vis. Fl. Dalm. III, p. 70 (1852).

Sporaden: Insel Jura (Reiser). Insel Tragonisi im Golfe von Petali (Holzmann). Attica: auf dem Pentelicon (Heldreich). Argolis: Hügel gegenüber Poros (Heldreich).

83. Lonicera implexa Ait. Hort. Kew, I, p. 231 (1789).

Attica: auf dem Pentelicon bei Pikermi (Heldreich). Sporaden: Insel Psathura (Reiser).

84. Rubia peregrina L. Sp. pl. p. 109 (1753).

Cycladen: Insel Naxos (Reiser).

1) Von anderen teratologischen Bildungen, welche mir bej Umbelliferenfüuchten theils withread dur Beobachtung theils gelegentlich der Literaturdurchsicht auffielen, sei nur noch eine die Frucht betreffende angeführt, welche nicht ganz selten vorzukommen scheint, aber bei Penzig (l. c) für keine Art erwähnt wird. Fs fehlt nämlich znweilen die dorsal in der Mediane gelegene Rippe der Frucht, infolge dessen natülich auch das unter der Rippe gelegene Gefässbündel; das Perientp weist vielmehr dorsal in der Mediane cinen Secretgang, eine "vitta auf. So bei Selinum Carvifolia (cf. Reichenbach l. c. Tafel 101), bei Scandix pecten Veneris (cf. Moynier de Villepoix, Ann. d. sc. nat., S. VI, t. 5. 1878). Ich fand das Gleiche bei Archangelica litoralis und bei Anthriscus silvestris an je einer Frucht.

") Vergl. Nr. 7, S. $25 \%$. 